\title{
A betabaculovirus encoding a gp64 homolog
}

Daniel M P. Ardisson-Araújo ${ }^{1 \dagger}$, Bruna T. Pereira ${ }^{2 \dagger}$, Fernando L. Melo', Bergmann M. Ribeiro', Sônia N. Báo', Paolo M. de A. Zanotto ${ }^{3}$, Flávio Moscardi ${ }^{4}$, Elliot W. Kitajima ${ }^{5}$, Daniel R. Sosa-Gomez ${ }^{4}$ and José L. C. Wolff ${ }^{6^{*}}$

\begin{abstract}
Background: A betabaculovirus (DisaGV) was isolated from Diatraea saccharalis (Lepidoptera: Crambidae), one of the most important insect pests of the sugarcane and other monocot cultures in Brazil.

Results: The complete genome sequence of DisaGV was determined using the 454-pyrosequencing method. The genome was 98,392 bp long, which makes it the smallest lepidopteran-infecting baculovirus sequenced to date. It had a G + C content of $29.7 \%$ encoding 125 putative open reading frames (ORF). All the 37 baculovirus core genes and a set of 19 betabaculovirus-specific genes were found. A group of 13 putative genes was not found in any other baculovirus genome sequenced so far. A phylogenetic analysis indicated that DisaGV is a member of Betabaculovirus genus and that it is a sister group to a cluster formed by ChocGV, ErelGV, PiraGV isolates, ClanGV, CaLGV, CpGV, CrleGV, AdorGV, PhopGV and EpapGV. Surprisingly, we found in the DisaGV genome a G protein-coupled receptor related to lepidopteran and other insect virus genes and a gp64 homolog, which is likely a product of horizontal gene transfer from Group 1 alphabaculoviruses.

Conclusion: DisaGV represents a distinct lineage of the genus Betabaculovirus. It is closely related to the CpGV-related group and presents the smallest genome in size so far. Remarkably, we found a homolog of gp64, which was reported solely in group 1 alphabaculovirus genomes so far.
\end{abstract}

\section{Background}

Brazil is the largest sugarcane (Saccharum officinarum, L.) and bioethanol producer in the world [1, 2]. Nowadays, sugarcane is grown on an area over 8 million hectares for both sugar and alcohol production [2]. As with other cultures cultivated over large areas, pest control is of crucial importance. The sugarcane borer Diatraea saccharalis Fabr. (Lepidoptera: Crambidae) is present in all sugarcaneproducing regions of the country, and is considered the major sugarcane pest, especially in the Southeast region [3]. Biological control based on the release of the parasitoid Cotesia flavipes (Cameron) (Hymenoptera: Braconidae) has been used with success in the control of the sugarcane borer $[4,5]$. However, other complementary and

\footnotetext{
* Correspondence: joseluiz.wolff@mackenzie.br

${ }^{\dagger}$ Equal contributors

'Deceased

${ }^{6}$ Laboratório de Biologia Molecular e Virologia, Centro de Ciências Biológicas e da Saúde (CCBS), Universidade Presbiteriana Mackenzie, São Paulo, SP, Brazil Full list of author information is available at the end of the article
}

compatible methods, such as the application of baculoviruses, would be highly desirable.

Baculoviruses are a large group of insect-specific viruses with circular double-stranded DNA, whose hallmark is the presence of occlusion bodies (OBs) [6]. The family Baculoviridae comprises four genera: two of them, Alphabaculovirus and Betabaculovirus, infect insects of the order Lepidoptera; the other two Gammabaculovirus and Deltabaculovirus infect insects of the orders Hymenoptera and Diptera [7] respectively. To date, the genomes of 73 baculovirus species were completely sequenced, and 17 of them are betabaculoviruses.

The baculovirus from the species Anticarsia gemmatalis multiple nucleopolyhedrovirus (AgMNPV) has been used in Brazil in one of the largest biocontrol programs in the world to control an insect pest [8]. Other successful programs with baculoviruses have been reported elsewhere in the world [6]. The success of the AgMNPV program is due to a combination of factors, such as: high virulence, dead larvae can be collected directly from the field to be used as inoculum, efficient application technology, etc. 
Nevertheless, development is needed on pest species that are not so easily exposed to the virus, as in the case of borers. Large-scale DNA sequencing provides information on complete viral genomes allowing for "omic" approaches that will eventually facilitate the development of application strategies. Since Brazil has high biodiversity, several baculoviruses have been found and their genomes sequenced [9-14]. With this prospect in mind, we have sequenced and analyzed the genome of a Diatraea saccharalis granulovirus (DisaGV), the first betabaculovirus isolated from a member of the family Crambidae. The presence of a functional gp64 homolog [15] in a betabaculovirus was a unique and remarkable finding.

\section{Results and discussion}

\section{Viral infection confirmation}

Larvae of Diatraea saccharalis with virus infection symptoms were found in sugarcane fields in the Southern Brazil. We performed the structural characterization of the putative virus and a granulovirus infection was confirmed by transmission electron microscopy of OBs extracted from larvae cadavers. Each elliptical granule had a single rod-shaped virion surrounded by a robust protein matrix coat (Fig. 1), indicating the typical morphology of granuloviruses (GVs) [6]. Since the protein matrix is formed by granulin produced in large amounts during late infection and because it is highly conserved among lepidopteran-infective baculovirus, we amplified and sequenced the granulin gene in order to obtain an initial confirmation to the viral type (data not shown). The 747 bp length of the DisaGV granulin had high amino acid identity with orthologs from the genus Betabaculovirus

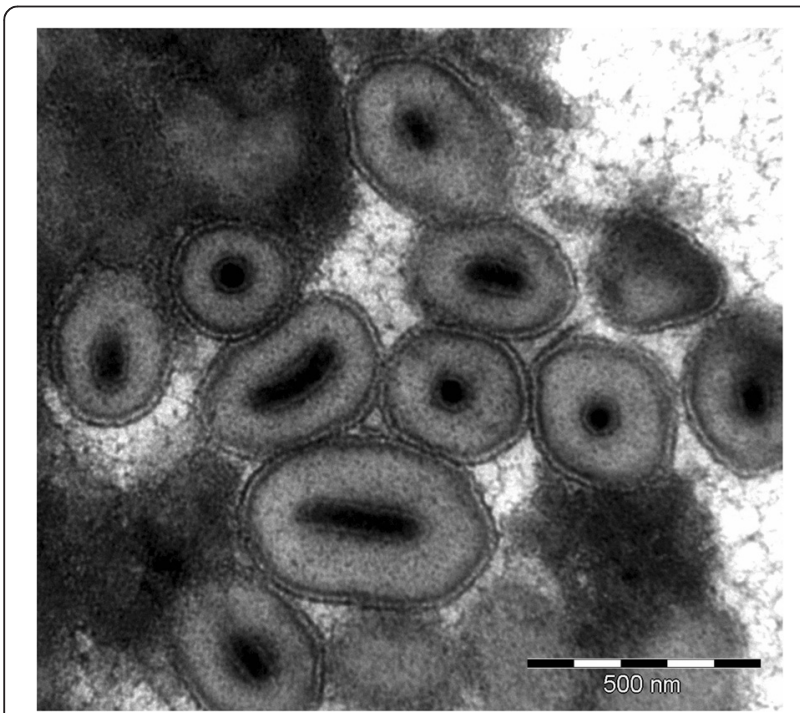

Fig. 1 Ultrastructural analysis of Diatraea saccharalis granulovirus (DisaGV). Transmission electron micrograph reveals granular occlusion bodies containing singly embedded rod-shaped nucleocapsid (scale bars $=0.5 \mu \mathrm{m}$ ) (data not shown). Importantly, a granulovirus was previously isolated from $D$. saccharalis caterpillars sampled from sugarcane crops in the southern United States. However, there is no available sequence to establish any phylogenetic relationship to the Brazilian strain described here [16].

\section{DisaGV genome and phylogeny}

The complete genome of DisaGV (Genbank accession number: KP296186) was 98,392 bp in length (mean coverage of $36 \mathrm{x}$ ), which makes the DisaGV the smallest betabaculovirus sequenced to date, followed by Adoxophyes orana granulovirus (AdorGV) $(99,657 \mathrm{bp})$ [17] and Plutella xylostella granulovirus (PlxyGV) (100,999 bp) [18]. The $G+C$ content was $29.7 \%$, a typically low value found among GVs, and potentially encoded 125 open reading frames (ORFs) with at least 50 predicted amino acid residues (Additional file 1: Table S1 and Fig. 2). The current baculovirus proposed species demarcation criterion is based on pairwise nucleotide distances estimated using the Kimura 2-parameter model of nucleotide substitution for three genes, granulin, lef-8, and lef-9 [7]. The pairwise distances of the viral sequences of DisaGV to other betabaculoviruses for both single loci and concatenated alignment are well in excess of 0.05 substitutions/site, fulfilling all the criteria for a novel species (data not shown). In order to investigate the phylogenetic relationship of DisaGV to other baculoviruses, we carried out a maximum likelihood phylogenetic analysis based on the alignment of the 37 baculovirus core proteins from all baculovirus genomes publicly available using solely the unique species (Additional file 2: Table S2). In agreement with both $\mathrm{OB}$ ultrastructural analysis and granulin gene sequencing (data not shown), we found DisaGV as sister taxa of the cluster formed by Choristoneura occidentalis granulovirus (ChocGV), Erinnyis ello granulovirus (ErelGV), Pieris rapae granulovirus (PiraGV) isolates, Clostera anachoreta granulovirus (ClanGV), Clostera anastomosis granulovirus (CaLGV), Cydia pomonella granulovirus (CpGV) and Cryptophlebia leucotreta granulovirus (CrleGV) (Fig. 3a).

Moreover, we performed a genomic comparison among some selected betabaculovirus genomes by MAUVE analysis. We found nine Locally Collinear Blocks (LCB), composed of genomic segments that appear to have the same relative position of their shared genes (Fig. 3b). Interestingly, LCB5 (from bp 20013 to 37032), LCB7 (from bp 40326 to 76348 ) and LCB8 (from bp 76601 to 87652 ) had an unexpected gene content composition. LCB5 lacked baculovirus core genes $\left(\chi^{2}=2.46, p<0.05\right.$, df $\left.=3\right)$, while LCB7 had a higher than expected number $\left(\chi^{2}=3.84, p<\right.$ 0.05 , df $=3$ ) and LCB8 had a higher than expected number of DisaGV-unique genes, a lower than expected number of baculovirus core genes and less than expected GVspecific genes $\left(\chi^{2}=5.12, p<0.01, \mathrm{df}=3\right)$. 


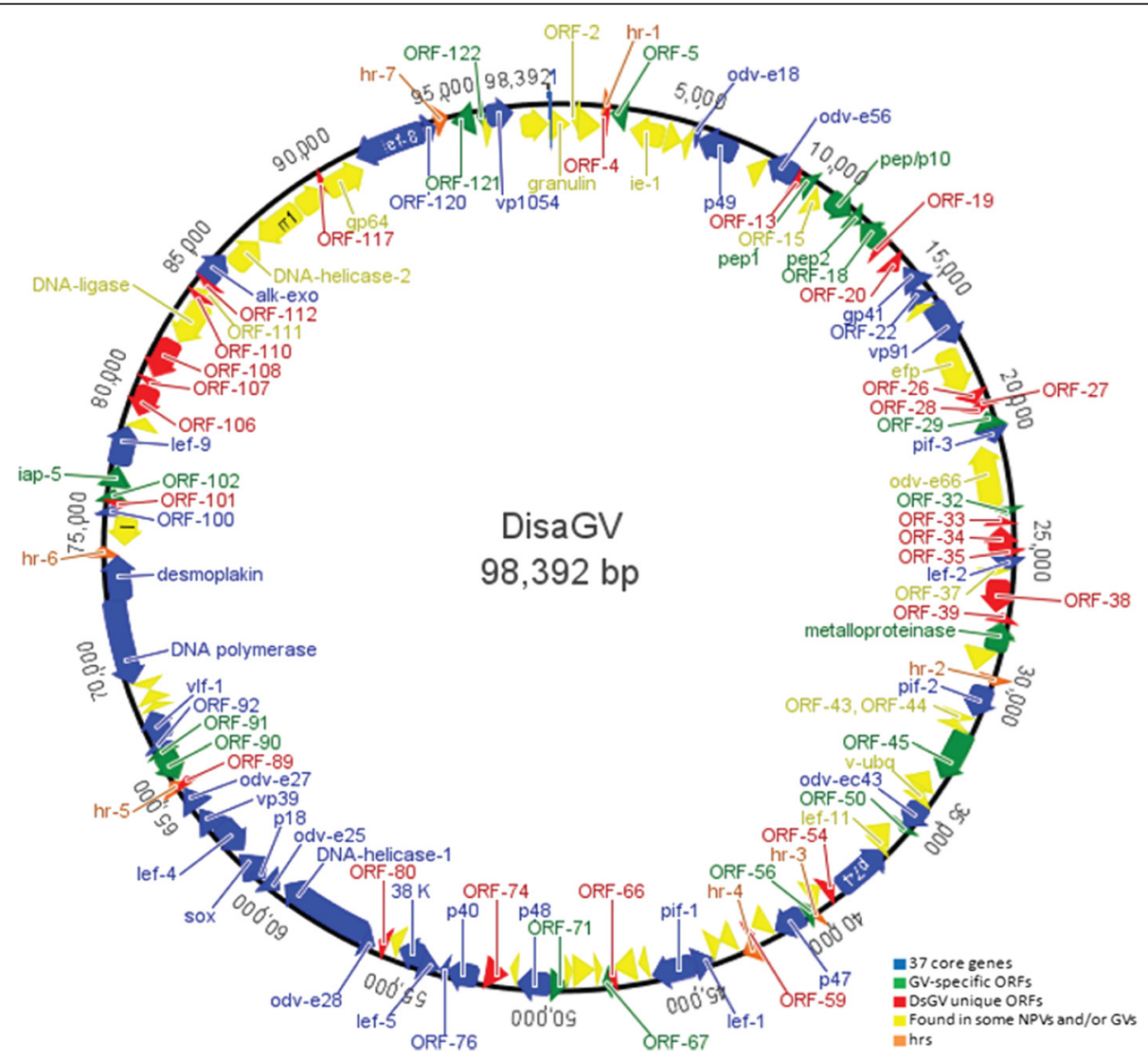

Fig. 2 Circular genome map of DisaGV with all genes identified on the 98,392 bp long. Arrows show the transcripcional orientation and relative size of each ORF. Those are colored according to their presence into baculovirus genera: in blue the 37 core genes, in green only betabaculovirusspecific genes, in red the DisaGV unique genes, in yellow genes found in some subjects of both alpha and betabaculovirus, and homologous regions (hrs) in orange

DisaGV had a dna-ligase (disa107) and two helicase genes (helicase-1, disa081 and helicase-2, disa111) probably involved in replication, repair, and recombination of DNA [19]. We also identified a deoxyuridine triphosphatase (dut) gene (disa073) and the ribonucleotide reductase subunits $r r 1$ (disa112) and $r r 2 a$ (disa113), involved in nucleotide metabolism. Nevertheless, the role of those genes during baculovirus infection is not clear. It was noteworthy the absence of several genes for early transcription factors, such as the $i e-0, i e-2$, and pe38. There were also no similar sequences to the baculovirus repeated ORFs (bro genes), to the ecdysteroid UDPglucosyltransferase (egt), to the apoptosis inhibitor p35, and also to the cathepsin and chitinase genes. We observed that the egt gene was absent only in the genomes of four other GVs, Helicoverpa armigera granulovirus (HearGV), Pseudaletia unipuncta granulovirus (PsunGV), Spodoptera litura granulovirus isolate K1 (SpliGV-K1) and Xestia c-nigrum granulovirus (XecnGV), that form a distinct phylogenic cluster. On the other hand, the $p 35$ gene was found only in the genomes of ChocGV, CaLGV, ClanGV (Data not shown). The absence of the cathepsin and chitinase genes may be compensated by the presence of the putative gene for matrix metalloproteinase (a stromelysin-1-like gene, disa040). Whereas the loss of the cathepsin and chitinase genes is a common event among the betabaculoviruses [10], the matrix metalloproteinase gene is present in all betabaculoviruses sequenced to date [20]. The expression of a functional CpGV-encoded metalloproteinase into the Autographa californica multiple nucleopolyhedrovirus (AcMNPV) genome enhanced the virus virulence, promoted larval melanization, and could partially substitute for the viral cathepsin [21].

\section{DisaGV unique genes}

Homologs to 25 DisaGV ORFs were not found in the genome of other baculoviruses. Taking into account the $450 \mathrm{bp}$ region upstream of each unique ORF, three of them presented no previously characterized promoter motifs, 12 contained exclusively early promoter motifs (TATAW, TATAWAW, TATAWTW with $\mathrm{W}=\mathrm{A}$ or $\mathrm{T}$ ), and ten had both early and late (A/TTAAG) motifs (Additional file 1: Table S1). Two unique ORFs, disa034 and disa039 showed significant BlastP hits to other 


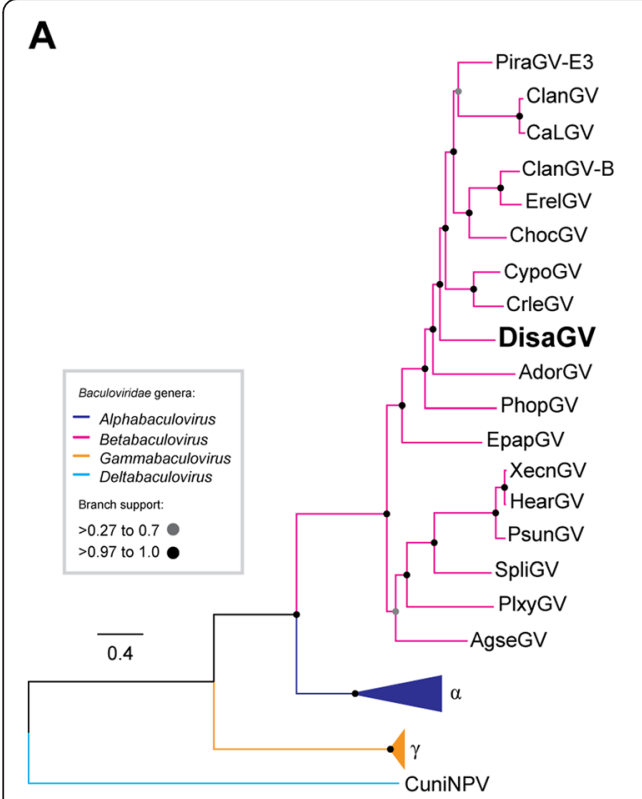

B

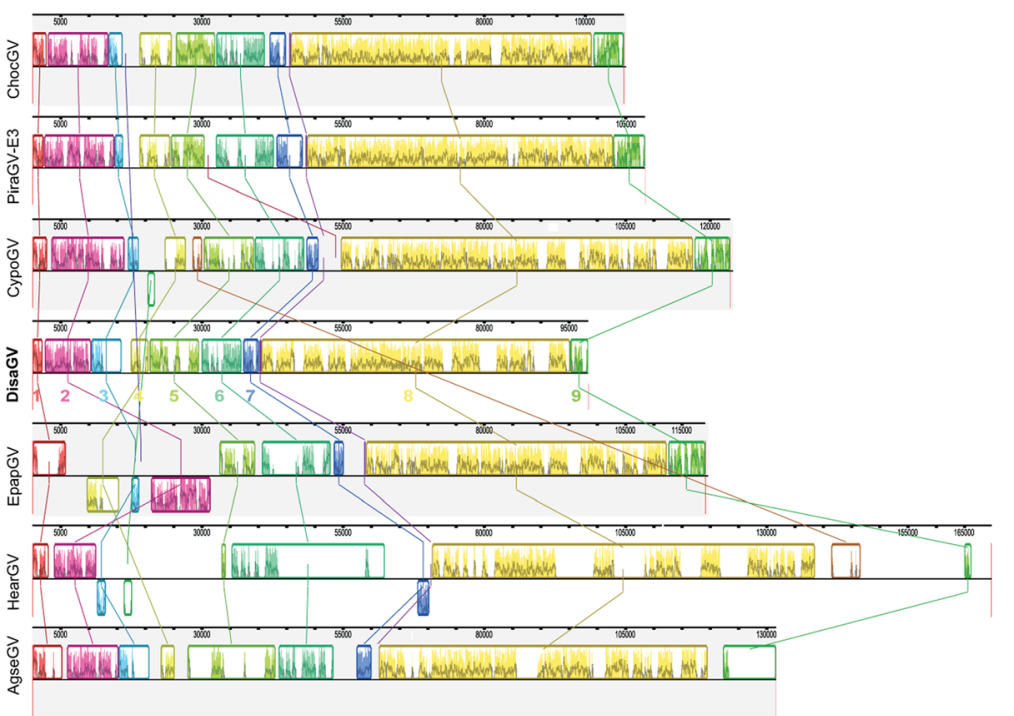

Fig. 3 Maximum-likelihood tree for Betabaculovirus and genome comparison. a The phylogeny was based on the concatenated amino acid sequences of the 37 core proteins identified in all baculovirus genome completely sequenced so far (Additional file 2: Table S2). We collapsed gammabaculoviruses (orange, $\gamma$ ) and alphaphabaculoviruses (dark blue, a). The CuniNPV was used as root (light blue). DisaGV (boldface) is a betabaculovirus and sister species of the cluster formed by CpGV-related species. b Genome comparison of the DisaGV genome against some related species including AgseGV, ChocGV, CpGV, EpapGV, and ErelGV. Locally collinear blocks (LCB) are numbered in the DisaGV genome from 1 to 9. Same colors depict same LCBs across the genomes. Rearrangement can be seen among the species

dsDNA virus sequences publicly available. ORF disa034 encoded a putative 310 aa protein that showed $26 \%$ amino acid identity (e-value $=1 \mathrm{e}-06$ ) to a 247 aa length protein of a phycodnavirus (Feldmannia irregularis virus a, AAR26869) (Fig. 4a). By motif search using the Pfam database [22], we found a conserved domain related to a PD-(D/E)XK nuclease superfamily, a highly diverse class of proteins. The exact biological functions, substrate specificity and molecular mechanisms of reactions for those nucleases remain unknown. However, quite a few enzymes are thought to be related to many cell process including replication, restriction, DNA repair and tRNAintron splicing [23]. Moreover, ORF disa039 coded for a hypothetical protein related to insect-infecting dsDNA
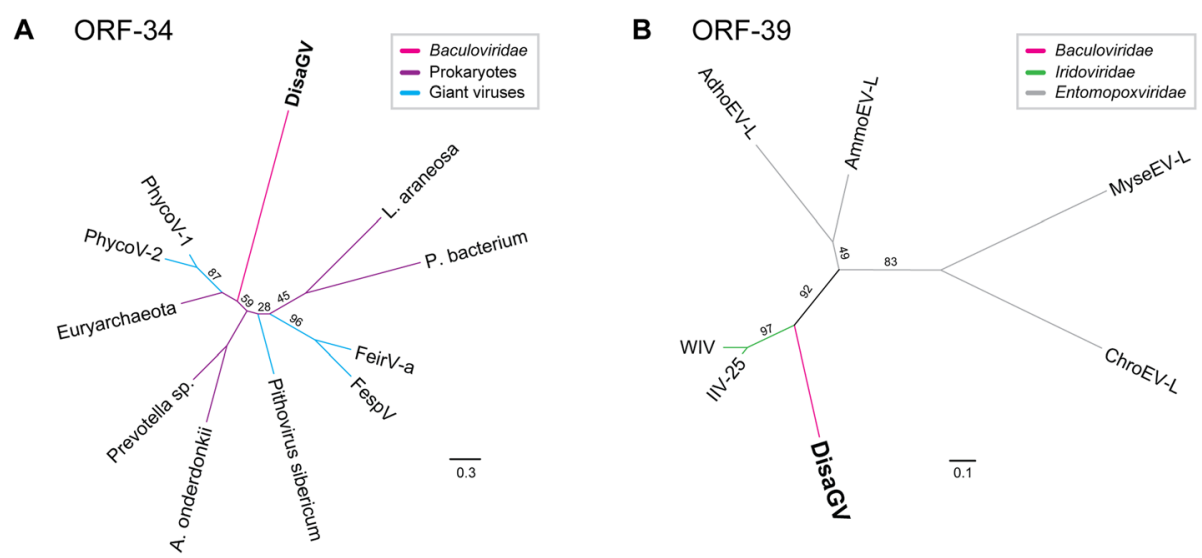

Fig. 4 Maximum likelihood phylogenetic trees of both Disa034 (a) and Disa039 (b) based on their predicted amino acid sequence. We used the RaxML method under the LG + I $+G$ model for Disa034 and WAG + I F for Disa039 with a nonparametric bootstrap to support the branches. Organisms: (a) Organic Lake phycodnaviruses (PhycoV-1 and PhycoV-2), Feldmannia species virus (FespV), Feldmannia irregularis virus a (FeirV-a) and Prokaryotes. b Wiseana iridescent virus (WIV), Invertebrate iridovirus 25 (IIV-25), Amsacta moorei entomopoxvirus 'L' (AmmoEV-L), Adoxophyes honmai entomopoxvirus 'L' (AdhoEV-L), Mythimna separata entomopoxvirus 'L' (MyseEV-L) and Choristoneura rosaceana entomopoxvirus 'L' (ChroEV-L) 
viruses including Wiseana iridescent virus (WIV) (YP 004732905, 131 aa) and Amsacta moorei entomopoxvirus 'L' (NP_064857, 158 aa) (Fig. 4b). Phycodnaviruses are eukaryotic algae viruses and seem to share a common ancestor with other insect dsDNA viruses, including iridoviruses and entomopoxviruses, which share baculovirus genes as well. Several baculovirus genes were found into the genome of those viruses, suggesting the occurrence of lateral gene transfer during co-infection in the same insect host, as probably expected to disa034 and disa039 [24].

\section{G protein-coupled receptor (GPCR)}

We also found another unique ORF (disa038) encoding a polypeptide related to a putative class $\mathrm{B}$ secretin-like G-protein coupled receptor (GPCR) of lepidopteran and an entomopoxvirus (Fig. 5a). GPCRs are cell

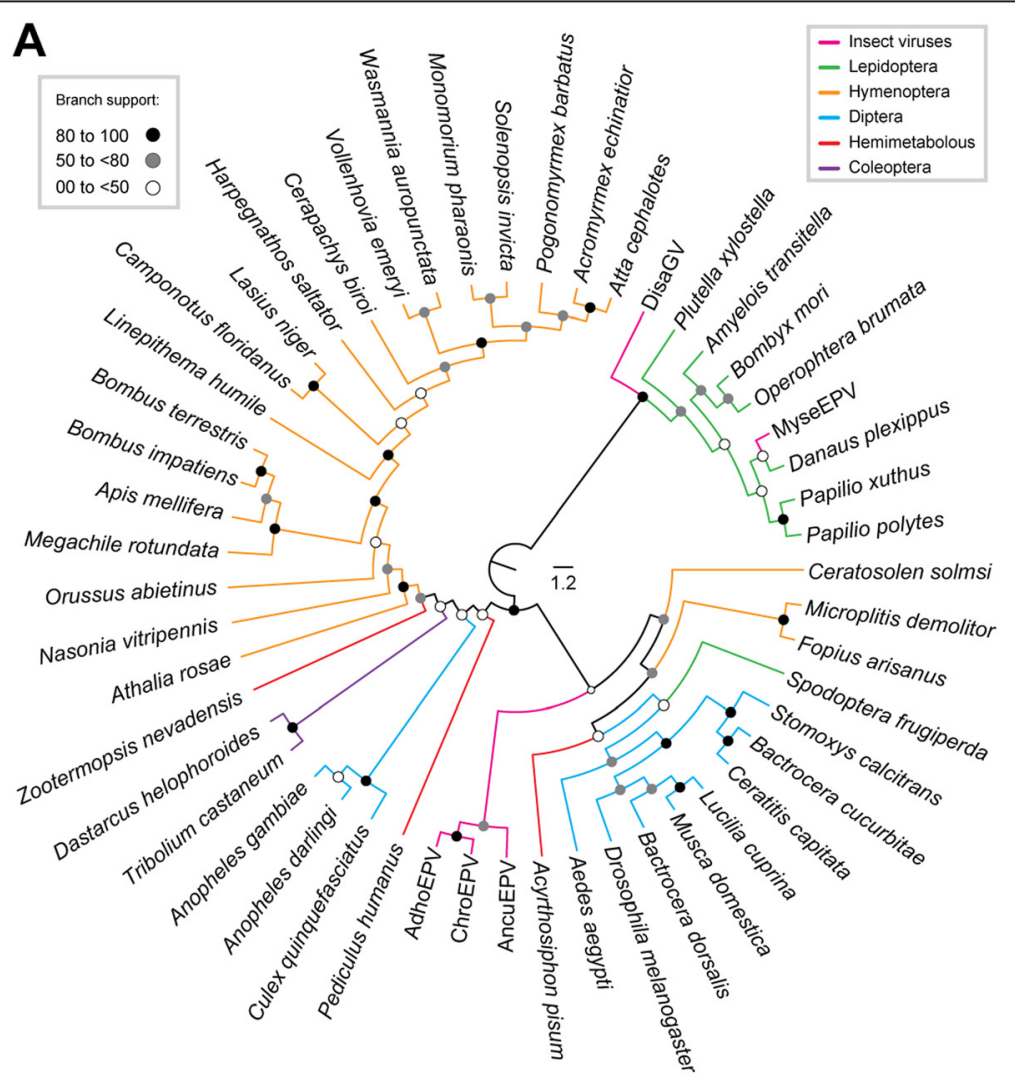

B

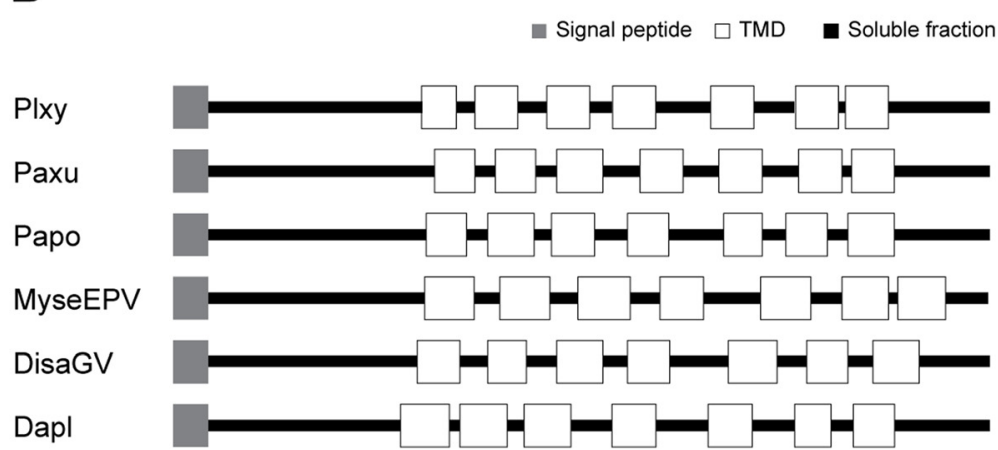

Fig. 5 In silico analyses of Disa038, a betabaculovirus-encoded G protein-coupled receptor gene. a Phylogenetic analysis of selected arthropod GPCRs. Disa038 sequence clustered with lepidopteran and an entomopoxvirus proteins. We performed the RaxML method under the WAG $+I+G+F$ model with a nonparametric bootstrap. The tree is presented as a cladogram. b Schematic representation of Disa038 and phylogenetically related proteins. The betabaculovirus GPCR retained all the structures observed in the homologs including the signal peptide (gray), soluble fraction (black), and the transmembrane domains (TMDs, white). Plxy, Plutella xylostella; Psxu, Papilio xuthus; Papo, Papilio polytes; MyseEV, Mythimna separata entomopoxvirus 'L'; and Dapl, Danaus plexippus 
membrane-associated GTPases that transmit signals from the environment to the cell inside or between cells. This allows them to react to a corresponding variety of extracellular stimuli that can be mediated by different peptides, lipids, proteins, nucleotides, nucleosides, organic odorants and photons [25]. This type of receptor has been described in many animal species despite not being quite common in virus genomes. We found a predicted signal peptide and seven trans-membrane domains in Disa038 (Fig. 5b), which strongly suggests that it is a member of the Secretin family [26]. Three subfamilies are recognized for this family and one of them, the B2 contains receptors with long extracellular N-termini as observed for both the predicted Disa038 and the other related proteins. It is not clear what role of this gene plays in the DisaGV life cycle. Nevertheless, it is noteworthy that human herpesvirus uses virally encoded GPCR to hijack cellular signaling network, which suggests a similar mechanism in DisaGV [27].

\section{GP64}

The most striking finding in the DisaGV genome was the presence of a gp64 homolog gene, disa118. GP64 is the major envelope fusion protein (EFP) exclusively found in Group I alphabaculoviruses (G1- $\alpha$ ) [6]. Both Group 2 alphabaculovirus $(\mathrm{G} 2-\alpha)$ and betabaculovirus share an analog to the GP64, called F protein, as the major budded virus (BV) EFP [28] which is probably the ancestral EFP in baculovirus [7, 29]. GP64 was acquired probably later by the ancestor of G1- $\alpha$ likely from an insect retrovirus-like element $[30,31]$ and is clearly related to the glycoprotein found in the genus Thogotovirus (from Orthomyxoviridae, an ssRNA negative-strand segmented virus family) [32]. Therefore, in attempt to understand both acquisition and evolution of gp64 into the DisaGV genome, we performed a phylogenetic reconstruction of the gene. We found that DisaGV GP64 clustered with G1- $\alpha$ EFP, suggestive of a horizontal gene transfer (HGT) from G1- $\alpha$ to betabaculovirus (Fig. 6a). Disa-GP64 clustered with Dendrolimus kikuchii nucleopolyhedrovirus (DekiNPV). Therefore, gp64 gene acquisition probably had adaptive value for the ancestor of DisaGV as it had for G1- $\alpha$. Taken together, these results suggest that the common ancestor of the G1- $\alpha$ acquired this gene once by HGT from some unknown source, which was later transferred to DisaGV or some related ancestral betabaculovirus. Alternatively, the gene was firstly acquired by a DisaGV-related virus and later

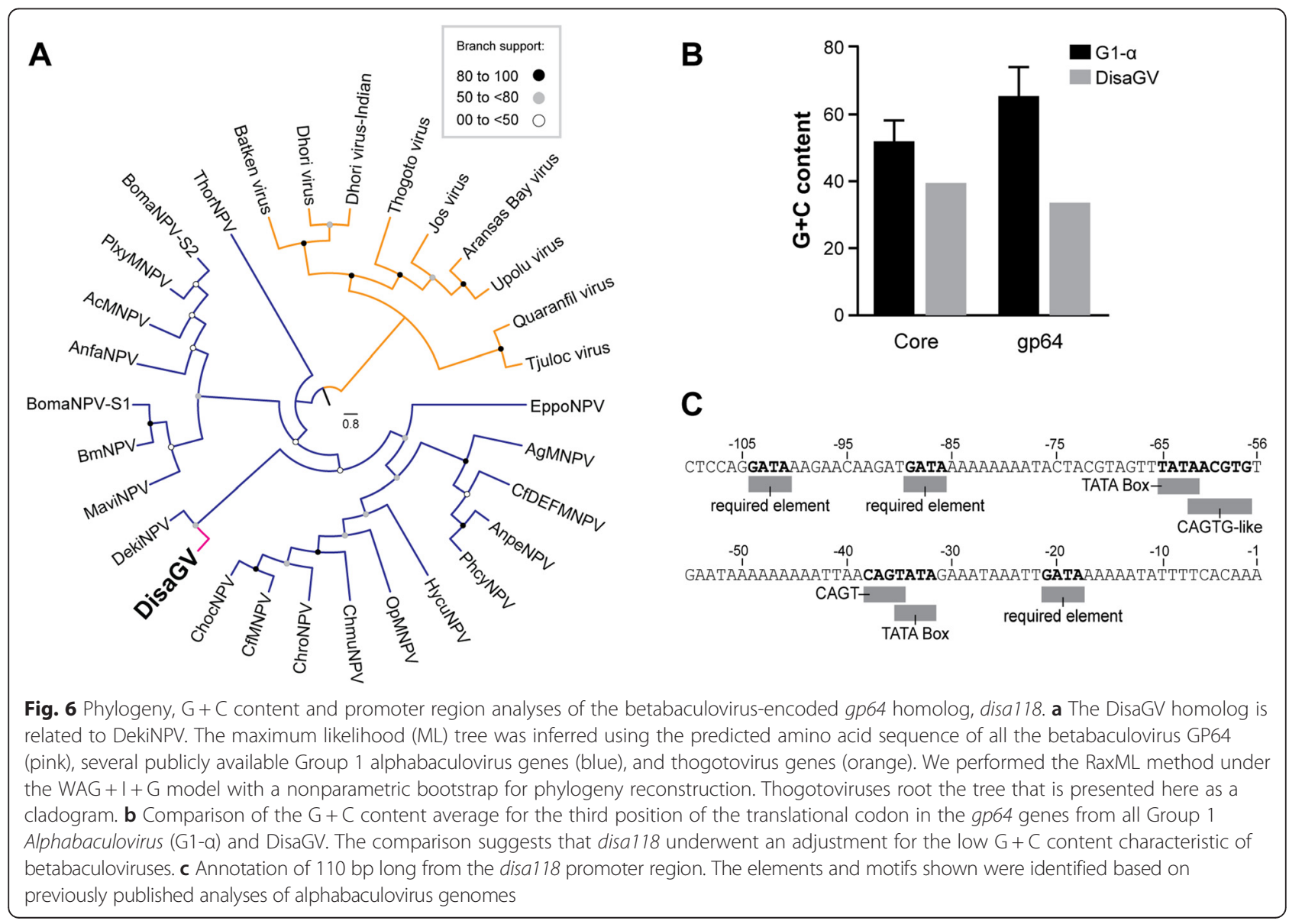


transferred to the common ancestor of G1- $\alpha$. An adaptation of disa118 to the $\mathrm{G}+\mathrm{C}$ genome content of DisaGV was observed (Fig. 6b) depicting that the gene acquisition is likely not recent [33]. Experimental analysis has shown that the incorporation of GP64 into the genome of Helicoverpa armigera nucleopolyhedrovirus (HaNPV), a G2 $\alpha$-baculovirus, enhanced virus infectivity in vivo and in vitro [34]. GP64 and F protein can exploit either distinct [35] or similar [36] receptors to entry into host cells. Therefore, gp64 acquisition has probably enhanced both fusion and binding virus capabilities [37, 38] and possibly replaced functionally the F protein in G1- $\alpha$ [30]. This evolutionary replacement hypothesis is reinforced by the fact that G1- $\alpha$ encode a remnant F protein homo$\log$ in their genomes that is unable to compensate for gp64 loss, albeit probably playing a role in the virus pathogenicity [39]. Interestingly, despite the fact that the DisaGV genome encodes an $\mathrm{F}$ protein, large deletions were observed in several reads covering the gene, suggesting existence of genotypes with deleted segments in the sequenced population (data not shown). This feature may indicate that the function of $f$ protein has been replaced or complemented by gp64 in DisaGV. Moreover, we also inspected the 150 nucleotides up-stream from the predicted DisaGV gp64 ATG start codon and compare with several G1 $\alpha$-baculovirus gp64 promoter regions (Fig. 6c). During viral de novo synthesis, gp64 expression is regulated by transcription from both early and late promoters with negative and multiple positive regulatory elements [40]. The gp64 promoter region size was previously described to be around 140 bp [41-43]. In the DisaGV, we found three required elements GATA $(-21,-89$, and -104$), 2$ TATA Box-like (-35 and -76$), 2$ CACGTG-like ( -38 and -61$)$ sequences with mutation on the first $\mathrm{C}$ to $\mathrm{A}$ in both, and one TATA-box (-35)-associated CAGT (-38). It is noteworthy that TATAdependent activity and TATA-independent activity is mediated by RNA polymerase II in OpMNPV gp64 [44], where two of the required GATA and CACGTG specifically bind to host transcription factors and activate transcription from the TATA-dependent gp 64 promoter $[44,45]$. The presence of these conserved regulatory expression sequences in the promoter region of disa-gp64 gene indicates that it must be transcribed and functional. In a recently published work, we found that disa-gp64 is a functional envelope fusion protein able to replace the native AcMNPV gp64 in a null recombinant baculovirus [15].

\section{Conclusion}

After structural characterization, complete genome sequence, and phylogenetic analyses of the Diatraea saccharalis-infecting virus (DisaGV), we found it to be a novel, distinct lineage of the Betabaculovirus genus. The genome seemed to be closely related to CpGV-related group but had the smallest genome found among other betabaculoviruses so far. Its genome encoded a GPCR-like gene and, remarkably for a functional gp64 gene, which had been previously reported solely for group 1 alphabaculovirus genomes.

\section{Methods}

\section{Viral origin, confirmation, and electron microscopy}

The DisaGV used in this study was obtained from infected larvae $D$. saccharalis collected in the state of Parana, Brazil in 2009. Transmission electron microscopy (TEM) of purified OBs and granulin gene amplicon sequence confirmed that the infection was due to a betabaculovirus. The granulin amplification was performed with universal primers for the major $\mathrm{OB}$ protein gene as previously published [46]. The amplified fragment was purified from an agarose gel after electrophoresis with the GFX kit (GE Healthcare) following the manufacturer's instructions, Sanger sequencing reaction was performed with the BigDye kit (Applied Biosystems) and the sequence determined in an automated sequencer ABI Prism 3100 Genetic Analyzer (Applied Biosystems). For transmission electron microscopy, a suspension of occlusion bodies extracted from larvae infected by DisaGV was prepared, as described elsewhere [10].

\section{Sequencing system, assembly, and analysis of the DisaGV complete genome}

DisaGV genomic DNA was sequenced with the 454 Genome Sequencer (GS) FLX ${ }^{\mathrm{Tw}}$ Standard (Roche) at the Centro de Genômica de Alto Desempenho do Distrito Federal (Brasília, Brazil). The genome was assembled de novo using Geneious 7.0 [47] and confirmed by comparing the restriction endonuclease fragment pattern from digestion of viral DNA to the fragment pattern generated from the assembled sequence. The annotation was performed using Geneious 7.0 to identify the ORFs that started with a methionine codon (ATG) encoding at least 50 amino acids and blastp to identify homologs. Specific primers were designed to amplify and sequence, by Sanger method, all regions in the genome with low coverage $(<10 \mathrm{x})$.

\section{Phylogenetic analyses and genome comparison}

For the Baculoviridae phylogeny, a MAFFT alignment [48] was carried out with the concatenated amino acid sequences predicted for the 37 baculovirus core genes. Phylogenetic inference was done using the FastTree method [49], implemented in Geneious. For evaluating putative HGTs events the same alignment method was used for Disa034, Disa038, Disa039 (GPCR-encoding gene), and Disa118 (gp64 homolog) and the phylogenetic tree was inferred using the RaxML program with 100 
replications of non parametric bootstrap [50], implemented in Geneious, with the models WAG $+\mathrm{I}+\mathrm{G}$ for GP64, $\mathrm{WAG}+\mathrm{I}+\mathrm{G}+\mathrm{F}$ for Disa038, WAG + I + F for Disa039, and $\mathrm{LG}+\mathrm{I}+\mathrm{G}$ for Disa034 selected by Prottest 2.4 [51]. The signal peptide and the transmembrane domains were predicted by both the SignalP 4.1 server (http:// www.cbs.dtu.dk/services/SignalP/) and the TMHMM Sever v. 2.0 (http://www.cbs.dtu.dk/services/TMHMM/), respectively. Moreover, the complete genome of DisaGV was compared with other betabaculovirus genomes through construction of syntenic maps with the Mauve program in the Geneious 7.1.7 using default parameter settings.

\section{Availability of supporting data}

The datasets of Figs. 3a, 4a, b, 5a and 6a supporting the conclusions of this article are available in the TreeBase [http://treebase.org/treebase-web/home.html] repository, [http://purl.org/phylo/treebase/phylows/ study/TB2:S18717].

\section{Additional files}

Additional file 1: Table S1. Gene content. (DOC $313 \mathrm{~kb}$ )

Additional file 2: Table S2. Virus isolates used in this paper for the reconstruction of the baculovirus phylogeny. (DOC $93 \mathrm{~kb}$ )

\begin{abstract}
Abbreviations
AcMNPV: Autographa californica multiple nucleopolyhedrovirus; AdorGV: Adoxophyes orana granulovirus; AgMNPV: Anticarsia gemmatalis multiple nucleopolyhedrovirus; bp: base pairs; BV: budded virus; CaLGV: Clostera anastomosis granulovirus; ChocGV: Choristoneura occidentalis granulovirus; ClanGV: Clostera anachoreta granulovirus; CpGV: Cydia pomonella granulovirus; CrleGV: Cryptophlebia leucotreta granulovirus;

DekiNPV: Dendrolimus kikuchii nucleopolyhedrovirus; DisaGV: Diatraea saccharalis granulovirus; EFP: envelope fusion protein; Egt: ecdysteroid UDPglucosyltransferase; ErelGV: Erinnyis ello granulovirus; G1-a: Group I alphabaculovirus; G2-a: Group II alphabaculovirus; GPCR: G-protein coupled receptor; GV: granulovirus; HaNPV: Helicoverpa armigera nucleopolyhedrovirus; HearGV: Helicoverpa armigera granulovirus; HGT: horizontal gene transfer; hr: homologous region; LCB: Locally Collinear Block; OB: occlusion body; ORF: open reading frame; PiraGV: Pieris rapae granulovirus; PIxyGV: Plutella xylostella granulovirus; PsunGV: Pseudaletia unipuncta granulovirus; SpliGVK1: Spodoptera litura granulovirus isolate K1; TEM: Transmission electron microscopy; WIV: Wiseana iridescent virus; XecnGV: Xestia c-nigrum granulovirus.
\end{abstract}

\section{Competing interests}

The authors declare that they have no competing interests.

\section{Authors' contributions}

BMR and JLCW conceived the project and designed the research experiments. BTP, DMPAA, FLM participated in the genome sequencing, assembly and annotation. DRSG, FM identified the diseased larvae and purified the granules. EWK prepared the transmission electron micrograph of the granules. DMPAA, FLM, PMAZ carried out the phylogenetic tree and synteny analysis. BTP, DMPAA, FLM, BMR, PMAZ, JLCW analyzed the data. BTP, DMPAA, FLM, BMR, PMAZ, JLCW wrote and revised the manuscript. BMR, SNB and JLCW provided research funds. All authors read and approved the final manuscript.

\section{Acknowledgements}

BTP received a scholarship from CAPES through the Graduate Program in Biotechnology from the University of São Paulo and Butantan Institute. This project was partially funded by CNPq grant 481741/2008-9. We thank Conselho Nacional de Desenvolvimento Científico e Tecnológico (CNPq) and Fundação de Apoio à Pesquisa do Distrito Federal (FAPDF).

\section{Author details}

'Departamento de Biologia Celular, Instituto de Ciências Biológicas, Universidade de Brasília, Brasília, DF, Brazil. ${ }^{2}$ Programa de Pós-graduação Interunidades em Biotecnologia, Instituto de Ciências Biomédicas (ICB), Universidade de São Paulo (USP), São Paulo, São Paulo, Brazil. ${ }^{3}$ Laboratório de Evolução Molecular e Bioinformática (LEMB-ICB), Universidade de São Paulo, São Paulo, SP, Brazil. ${ }^{4}$ Empresa Brasileira de Pesquisa Agropecuária, Centro Nacional de Pesquisa de Soja, Londrina, Paraná PR, Brazil. ${ }^{5}$ NAP/MEPA, Departamento de Fitopatologia e Nematologia, ESALQ, Universidade de São Paulo, Piracicaba, SP, Brazil. '́Laboratório de Biologia Molecular e Virologia, Centro de Ciências Biológicas e da Saúde (CCBS), Universidade Presbiteriana Mackenzie, São Paulo, SP, Brazil.

Received: 9 October 2015 Accepted: 20 January 2016

Published online: 04 February 2016

\section{References}

1. Zanin GM, Santana CC, Bon EP, Giordano RC, de Moraes FF, Andrietta SR, et al. Brazilian bioethanol program. Appl Biochem Biotechnol. 2000;84-86:1147-61.

2. Soccol CR, Vandenberghe LP, Medeiros AB, Karp SG, Buckeridge M, Ramos LP, et al. Bioethanol from lignocelluloses: Status and perspectives in Brazil. Bioresour Technol. 2010;101(13):4820-5.

3. Dinardo-Miranda LL. Pragas. In: Dinardo-Miranda LL, Vasconceles ACM, Landell MGA, editors. Cana-de-açúcar. Campinas: Instituto Agronômico; 2008.

4. Rossi GD, Salvador G, Consoli FL. The parasitoid, cotesia flavipes (cameron) (hymenoptera: braconidae), influences food consumption and utilization by larval diatraea saccharalis (f.) (lepidoptera: crambidae). Arch Insect Biochem Physiol. 2014;87(3):85-94

5. Mahmoud AM, De Luna-Santillana EJ, Rodriguez-Perez MA. Parasitism by the endoparasitoid, Cotesia flavipes induces cellular immunosuppression and enhances susceptibility of the sugar cane borer, Diatraea saccharalis to Bacillus thuringiensis. J Insect Sci. 2011;11:119.

6. Rohrmann GF. Baculovirus Molecular Biology, Third Edition [Internet] edn. Bethesda (MD): National Center for Biotechnology Information (US); 2013. Available from: http://www.ncbi.nlm.nih.gov/books/NBK49500/.

7. Jehle JA, Lange M, Wang H, Hu Z, Wang Y, Hauschild R. Molecular identification and phylogenetic analysis of baculoviruses from Lepidoptera. Virology. 2006;346(1):180-93.

8. Moscardi F. Assessment of the application of baculoviruses for control of Lepidoptera. Annu Rev Entomol. 1999;44:257-89.

9. Ardisson-Araujo DM, Melo FL, de Souza Andrade M, Brancalhao RM, Bao SN, Ribeiro BM. Complete genome sequence of the first non-Asian isolate of Bombyx mori nucleopolyhedrovirus. Virus Genes. 2014;49(3):477-84.

10. Ardisson-Araujo DM, de Melo FL, Andrade MD, Sihler W, Bao SN, Ribeiro BM, et al. Genome sequence of Erinnyis ello granulovirus (ErelGV), a natural cassava hornworm pesticide and the first sequenced sphingid-infecting betabaculovirus. BMC Genomics. 2014;15(1):856.

11. Oliveira JV, Wolff JL, Garcia-Maruniak A, Ribeiro BM, de Castro ME, de Souza ML, et al. Genome of the most widely used viral biopesticide: Anticarsia gemmatalis multiple nucleopolyhedrovirus. J Gen Virol. 2006;87(Pt 11): $3233-50$.

12. Wolff JL, Valicente FH, Martins R, Oliveira JV, Zanotto PM. Analysis of the genome of Spodoptera frugiperda nucleopolyhedrovirus (SFMNPV-19) and of the high genomic heterogeneity in group II nucleopolyhedroviruses. J Gen Virol. 2008:89(Pt 5):1202-11.

13. Craveiro SR, Inglis PW, Togawa RC, Grynberg P, Melo FL, Ribeiro ZM, et al. The genome sequence of Pseudoplusia includens single nucleopolyhedrovirus and an analysis of p26 gene evolution in the baculoviruses. BMC Genomics. 2015:16:127.

14. Ardisson-Araujo DM, Sosa-Gómez DR, Melo FL, Báo SN, Ribeiro BM. Characterization of helicoverpa zea single nucleopolyhedrovirus isolated in brazil during the first old world bollworm (noctuidae: helicoverpa armigera) nationwide outbreak. Virus Rev Res. 2015:20:4

15. Ardisson-Araujo DM, Melo FL, Clem RJ, Wolff JL, Ribeiro BM. A Betabaculovirus-Encoded gp64 Homolog Codes for a Functional Envelope Fusion Protein. J Virol. 2015;90:1668-1672. 
16. Pavan OHO, Boucias DG, Almeida LC, Gaspar JO, Botelho PSM, Degaspari N. Granulosis Virus of Diatraea saccharalis (DsGV): pathogenicity, replication and ultrastructure. In: Proceedings of the International Congress of the International Society of Sugar Cane Technologists (ISSCT '83): 1983; Havana, Cuba. 1983. p. 644-59.

17. Wormleaton S, Kuzio J, Winstanley D. The complete sequence of the Adoxophyes orana granulovirus genome. Virology. 2003;311(2):350-65.

18. Hashimoto Y, Hayakawa T, Ueno Y, Fujita T, Sano Y, Matsumoto T. Sequence analysis of the Plutella xylostella granulovirus genome. Virology. 2000;275(2): 358-72.

19. Kuzio J, Pearson MN, Harwood SH, Funk CJ, Evans JT, Slavicek JM, et al. Sequence and analysis of the genome of a baculovirus pathogenic for Lymantria dispar. Virology. 1999;253(1):17-34.

20. Ishimwe E, Hodgson JJ, Clem RJ, Passarelli AL. Reaching the melting point: Degradative enzymes and protease inhibitors involved in baculovirus infection and dissemination. Virology. 2015;479-480:637-49.

21. Ishimwe E, Hodgson JJ, Passarelli AL. Expression of the Cydia pomonella granulovirus matrix metalloprotease enhances Autographa californica multiple nucleopolyhedrovirus virulence and can partially substitute for viral cathepsin. Virology. 2015;481:166-78.

22. Finn RD, Bateman A, Clements J, Coggill P, Eberhardt RY, Eddy SR, et al. Pfam: the protein families database. Nucleic Acids Res. 2014;42(Database issue):D222-30.

23. Steczkiewicz K, Muszewska A, Knizewski L, Rychlewski L, Ginalski K. Sequence, structure and functional diversity of PD-(D/E)XK phosphodiesterase superfamily. Nucleic Acids Res. 2012;40(15):7016-45.

24. Iyer LM, Balaji S, Koonin EV, Aravind L. Evolutionary genomics of nucleocytoplasmic large DNA viruses. Virus Res. 2006;117(1):156-84.

25. Kochman K. Superfamily of G-protein coupled receptors (GPCRs)-extraordinary and outstanding success of evolution. Postepy Hig Med Dosw (Online). 2014; 68:1225-37.

26. Krishnan A, Almen MS, Fredriksson $R$, Schioth HB. The origin of GPCRs: identification of mammalian like Rhodopsin, Adhesion, Glutamate and Frizzled GPCRs in fungi. PLoS ONE. 2012;7(1):e29817.

27. Nijmeijer S, Leurs R, Smit MJ, Vischer HF. The Epstein-Barr virus-encoded G protein-coupled receptor BILF1 hetero-oligomerizes with human CXCR4, scavenges Galphai proteins, and constitutively impairs CXCR4 functioning. J Biol Chem. 2010;285(38):29632-41.

28. Garry CE, Garry RF. Proteomics computational analyses suggest that baculovirus GP64 superfamily proteins are class III penetrenes. Virol J. 2008:5:28

29. Jehle JA, Blissard GW, Bonning BC, Cory JS, Herniou EA, Rohrmann GF, et al. On the classification and nomenclature of baculoviruses: a proposal for revision. Arch Virol. 2006;151(7):1257-66.

30. Wang M, Wang J, Yin F, Tan Y, Deng F, Chen X, et al. Unraveling the entry mechanism of baculoviruses and its evolutionary implications. J Virol. 2014 88(4):2301-11.

31. Rohrmann GF, Karplus PA. Relatedness of baculovirus and gypsy retrotransposon envelope proteins. BMC Evol Biol. 2001;1:1.

32. Morse MA, Marriott AC, Nuttall PA. The glycoprotein of Thogoto virus (a tick-borne orthomyxo-like virus) is related to the baculovirus glycoprotein GP64. Virology. 1992;186(2):640-6.

33. Monier A, Claverie JM, Ogata H. Horizontal gene transfer and nucleotide compositional anomaly in large DNA viruses. BMC Genomics. 2007:8:456

34. Shen S, Gan Y, Wang M, Hu Z, Wang H, Deng F. Incorporation of GP64 into Helicoverpa armigera nucleopolyhedrovirus enhances virus infectivity in vivo and in vitro. J Gen Virol. 2012;93(Pt 12):2705-11.

35. Westenberg M, Uijtdewilligen P, Vlak JM. Baculovirus envelope fusion proteins F and GP64 exploit distinct receptors to gain entry into cultured insect cells. J Gen Virol. 2007;88(Pt 12):3302-6.

36. Wang M, Yin F, Shen S, Tan Y, Deng F, Vlak JM, et al. Partial functional rescue of Helicoverpa armigera single nucleocapsid nucleopolyhedrovirus infectivity by replacement of F protein with GP64 from Autographa californica multicapsid nucleopolyhedrovirus. J Virol. 2010;84(21):11505-14.

37. Liang C, Song J, Chen X. The GP64 protein of Autographa californica multiple nucleopolyhedrovirus rescues Helicoverpa armigera nucleopolyhedrovirus transduction in mammalian cells. J Gen Virol. 2005; 86(Pt 6):1629-35

38. Yu IL, Lin YC, Robinson JH, Lung O. Transduction of vertebrate cells with Spodoptera exigua multiple nucleopolyhedrovirus F protein-pseudotyped gp64-null Autographa californica multiple nucleopolyhedrovirus. J Gen Virol. 2009;90(Pt 9):2282-7.
39. Lung OY, Cruz-Alvarez M, Blissard GW. Ac23, an envelope fusion protein homolog in the baculovirus Autographa californica multicapsid nucleopolyhedrovirus, is a viral pathogenicity factor. J Virol. 2003;77(1):328-39.

40. Blissard GW, Rohrmann GF. Baculovirus gp64 gene expression: analysis of sequences modulating early transcription and transactivation by IE1. J Virol. 1991;65(11):5820-7.

41. Jarvis DL, Garcia Jr A. Biosynthesis and processing of the Autographa californica nuclear polyhedrosis virus gp64 protein. Virology. 1994;205(1): 300-13.

42. Garrity DB, Chang MJ, Blissard GW. Late promoter selection in the baculovirus gp64 envelope fusion protein gene. Virology. 1997;231(2):167-81.

43. Chen YR, Zhong S, Fei Z, Hashimoto Y, Xiang JZ, Zhang S, et al. The Transcriptome of the Baculovirus Autographa californica Multiple Nucleopolyhedrovirus in Trichoplusia ni Cells. J Virol. 2013;87(11):6391-405.

44. Kogan PH, Chen X, Blissard GW. Overlapping TATA-dependent and TATAindependent early promoter activities in the baculovirus gp64 envelope fusion protein gene. J Virol. 1995;69(3):1452-61.

45. Kogan PH, Blissard GW. A baculovirus gp64 early promoter is activated by host transcription factor binding to CACGTG and GATA elements. J Virol. 1994:68(2):813-22

46. Lange $\mathrm{M}$, Wang $\mathrm{H}$, Zhihong $\mathrm{H}$, Jehle JA. Towards a molecular identification and classification system of lepidopteran-specific baculoviruses. Virology. 2004;325(1):36-47

47. Kearse M, Moir R, Wilson A, Stones-Havas S, Cheung M, Sturrock S, et al. Geneious Basic: an integrated and extendable desktop software platform for the organization and analysis of sequence data. Bioinformatics. 2012;28(12): 1647-9.

48. Katoh K, Misawa K, Kuma K, Miyata T. MAFFT: a novel method for rapid multiple sequence alignment based on fast Fourier transform. Nucleic Acids Res. 2002;30(14):3059-66.

49. Liu K, Linder CR, Warnow T. RAxML and FastTree: comparing two methods for large-scale maximum likelihood phylogeny estimation. PLOS ONE. 2011; 6(11):e27731.

50. Guindon S, Dufayard JF, Lefort V, Anisimova M, Hordijk W, Gascuel O. New algorithms and methods to estimate maximum-likelihood phylogenies: assessing the performance of PhyML 3.0. Syst Biol. 2010;59(3):307-21.

51. Abascal F, Zardoya R, Posada D. ProtTest: selection of best-fit models of protein evolution. Bioinformatics. 2005;21(9):2104-5.

\section{Submit your next manuscript to BioMed Central and we will help you at every step:}

- We accept pre-submission inquiries

- Our selector tool helps you to find the most relevant journal

- We provide round the clock customer support

- Convenient online submission

- Thorough peer review

- Inclusion in PubMed and all major indexing services

- Maximum visibility for your research

Submit your manuscript at www.biomedcentral.com/submit
) Biomed Central 